\title{
Sensory Systems as Cybernetic Systems that Require Awareness of Alternatives to Interact with the World: Analysis of the Brain-Receptor Loop in Norwich's Entropy Theory of Perception
}

\author{
Lance Nizami \\ Boys Town National Research Hospital \\ Omaha NE, USA \\ nizamii2@aol.com
}

\begin{abstract}
Introduction \& Objectives: Norwich's Entropy Theory of Perception (1975 [1] -present) stands alone. It explains many firing-rate behaviors and psychophysical laws from bare theory. To do so, it demands a unique sort of interaction between receptor and brain, one that Norwich never substantiated. Can it now be confirmed, given the accumulation of empirical sensory neuroscience? Background: Norwich conjoined sensation and a mathematical model of communication, Shannon's Information Theory, as follows: "In the entropic view of sensation, magnitude of sensation is regarded as a measure of the entropy or uncertainty of the stimulus signal" [2]. "To be uncertain about the outcome of an event, one must first be aware of a set of alternative outcomes" [3]. "The entropy-establishing process begins with the generation of a [internal] sensory signal by the stimulus generator. This is followed by receipt of the [external] stimulus by the sensory receptor, transmission of action potentials by the sensory neurons, and finally recapture of the [response to the internal] signal by the generator" [4]. The latter "recapture" differentiates external from internal stimuli. The hypothetical "stimulus generators" are internal emitters, that generate photons in vision, audible sounds in audition (to Norwich, the spontaneous otoacoustic emissions [SOAEs]), "temperatures in excess of local skin temperature" in skin temperature sensation [4], etc. Method (1): Several decades of empirical sensory physiology literature was scrutinized for internal "stimulus generators". Results (1): Spontaneous photopigment isomerization ("dark light") does not involve visible light. SOAEs are electromechanical basilar-membrane artefacts that rarely produce audible tones. The skin's temperature sensors do not raise skin temperature, etc. Method (2): The putative action of the brain-and-sensory-receptor loop was carefully reexamined. Results (2): The sensory receptor allegedly "perceives", experiences "awareness", possesses "memory", and has a "mind". But those traits describe the whole human. The receptor, thus anthropomorphized, must therefore contain its own perceptual loop, containing a receptor, containing a perceptual loop, etc. Summary \& Conclusions: The Entropy Theory demands sensory awareness of alternatives, through an imagined brain-and-sensory-receptor loop containing internal "stimulus generators". But (1) no internal "stimulus generators" seem to exist and (2) the loop would be the outermost of an infinite nesting of identical loops.
\end{abstract}

Keywords - cybernetics, information theory, loop, receptor, uncertainty

\section{INTRODUCTION}

The Entropy Theory of Perception of K.H. Norwich and co-authors is a mathematical opus that, at last count, was spread over no fewer than 20 peer-reviewed papers, a book, and at least 20 abstracts. The theory had a preternatural flexibility, explaining many firing-rate behaviors and psychophysical laws, all purely from algebra. To do so, it demanded a unique sort of interaction between the sensory receptor and the brain. The explanation of that interaction was spread over two papers and a conference proceeding [3]-[5]. Norwich and his later co-authors never provided data to substantiate the hypothesized interaction. However, it may now be extensively checked thanks to a great accumulation of neuroscience data in the intervening years.

\section{NORWICH ET AL.'S ENTROPY THEORY OF PERCEPTION}

The Entropy Theory will first be synopsized for the unfamiliar reader. As noted, the theory was spread among many papers. The crux of the theory is that we perceive only when uncertain, and that perception ends when certainty is reached [6]-[8]. Shannon's Information Theory states that reduction of uncertainty equals gain of information [9], and as such, it is there that the Entropy Theory seeks an explanation for psychophysical phenomena [10]-[13]. Shannon stated that uncertainty is caused by an event having $\mathrm{n}$ possible outcomes. Knowing those outcomes, and their probabilities of occurrence $\left\{\mathrm{p}_{1}, \ldots, \mathrm{p}_{\mathrm{n}}\right\}$, allows the calculation of some useful quantities, as follows. Shannon stated that the information associated with probability $p_{i}$ is $-\log \left(p_{i}\right)$. Still following Shannon, the average information derived from observing the outcome of the event, the information that Norwich et al. called the "stimulus information" and named $\mathrm{I}_{\mathrm{S}}$, is

$$
I_{S}=-\sum_{i=1}^{n} p_{i} \log p_{i}
$$


[11], [14]. When logarithms are in base 2, the information is in binary information units, or "bits". In base e $(=2.71828 \ldots)$, information is in "natural units". Maximising $\mathrm{I}_{\mathrm{S}}[15, \mathrm{p} 56]$ leads to equal probabilities $\left\{\mathrm{p}_{1}=\mathrm{p}_{2}=\ldots=\mathrm{p}_{\mathrm{n}}\right\}$. Thus, for example, the information transmitted when a person familiar with dice observes the roll of a single, fair, six-sided die will be $-6(1 / 6 \log 1 / 6)=\log 6$, the actual number depending on the chosen base of the logarithm. With only one outcome, conversely, $\mathrm{I}_{\mathrm{S}}=-1 \log 1=0$. That is, we already knew the outcome of the event, so no new information was available. In the Entropy Theory, an outcome that is certain is not perceivable [3], [10\}, [11], [16]-[18]; after [9].

\section{THE ROLE OF THE SENSORY RECEPTOR}

According to Norwich et al. (but note that Shannon offered no view on this), information is transmitted to the sensory receptor (not by the receptor) when a stimulus is applied [19]. As Norwich notes, "The sensory receptor forms part of a channel, and the afferent neuron part of a metachannel ... The metachannel transmits in coded form the degree of uncertainty residing at the receptor about some quality of the stimulus" [4, p175] (also [3]). For example, in vision, the stream of photons approaching the eye is the channel [3]. Thus, the sensory afferent does not transmit information about the stimulus attribute (say, intensity), but about the uncertainty removed at the receptor upon exposure to the stimulus [20], [21]. "In the entropic view of sensation, magnitude of sensation is regarded as a measure of the entropy or uncertainty of the stimulus signal" [2, p806]. For a steady light, the activity of the optic nerve relays the rate at which the eye is becoming more certain about the light [17].

The receptor organ's degree of uncertainty is represented by the entropy, which Norwich et al. called $\mathrm{E}_{\mathrm{S}}$ or H (e.g. [11]). The entropy was calculated after Shannon as follows. Imagine a communications system that is transmitting and receiving symbols. Let $\mathrm{p}_{\mathrm{j}}(\mathrm{k})$ be the probability of transmission of symbol $\mathrm{k}$, given that symbol $\mathrm{j}$ has been received (see [22]). Then

$$
E_{S}=-\sum_{j} \sum_{k} p_{j}(k) \log p_{j}(k)
$$

The neuronal or psychophysical response, both called $\mathrm{F}$ [6], [10], [11], [19], [20], [23] is proportional to $E_{S}$. Information transmitted $\mathrm{I}_{\mathrm{t}}$ is given by

$$
\begin{aligned}
I_{t}=I_{S}-E_{S}= & -\sum_{k} p(k) \log p(k) \\
& +\sum_{j} \sum_{k} p_{j}(k) \log p_{j}(k) .
\end{aligned}
$$

The origins of the latter equation for psychophysical use are explained in [24].

\section{RECEPTOR TO BRAIN: THE PERCEPTUAL LOOP}

\section{A. The Need for Foreknowledge}

Norwich [3, p835] noted that "To be uncertain about the outcome of an event, one must first be aware of a set of alternative outcomes". That is, one must have foreknowledge. For example, to have foreknowledge of intensity, "the receptor must previously have perceived a number of stimulus intensities, so that it may now 'doubt' which of the possible intensities, is present" [3, p835]. But how does the sensory receptor know a priori the potential intensities of a stimulus? According to Norwich ([4, p179], [5, p537]; see also [11], on taste), "one could postulate that nature endows the receptor with innate uncertainty, which will re-appear spontaneously after the act of perception, and in this way obviate the need for an active component to perception". Norwich dismisses this idea as "unsatisfying" $[5, \mathrm{p} 537]$ and "less convincing" [4, p179] than the approach to now be reviewed.

\section{B. Establishment of Uncertainty at the Receptor by the Action of the Perceptual Loop}

In the Entropy Theory, each sensory receptor forms part of a perceptual loop containing stimulus-generating powers, to provide self-calibration into a state of uncertainty and hence perceptual readiness [5]. To explain: according to the Entropy Theory, reduction of uncertainty (entropy) is a passive process, whereas creating uncertainty in the first place, before the presentation of any stimulus, is an active process [5]. Norwich provided the example that when flipping a coin, prior examination of the coin (an active process) establishes the two possibilities (heads or tails) that give the observer's uncertainty, $\log _{2}(2)$ bits. This uncertainty initialises the receptor [4]; all entropy reduction involves this "motor-sensory conjunction". For a mechanoreceptor, self-calibration might occur by actual limb movement [14]. For a stretch receptor, the realm of weights that can be held is established by lifting different weights [4]. Generally, "you cycle any device through its range of outputs" [3, p835] and the receptor itself must remember these possibilities [3].

\section{The Need for "Internal Transmitters"}

According to the Entropy Theory, the passive component of the perceptual unit must acknowledge the active component. For example, the act of examining a coin (active) must cause neuronal activity (passive) and so must the lifting of different known weights [4]. The perceiving of the force of a lifted weight must involve a "muscular component which is capable of actively generating a force" [5, p538]. Vision must involve the internal generation of photons [5] of at least two intensities of light [4] by a photoemitter [3]; the photons are then sensed by the [passive] visual receptor [3]-[5]. Similarly, the sensor for "temperature in excess of skin temperature, must be capable of generating temperatures in excess of local skin temperature" ([4, p180]; see also [3], [5]). No mention was made of sensors for temperatures less than skin temperature, which, by implication, would generate temperatures lesser than local skin temperature. 


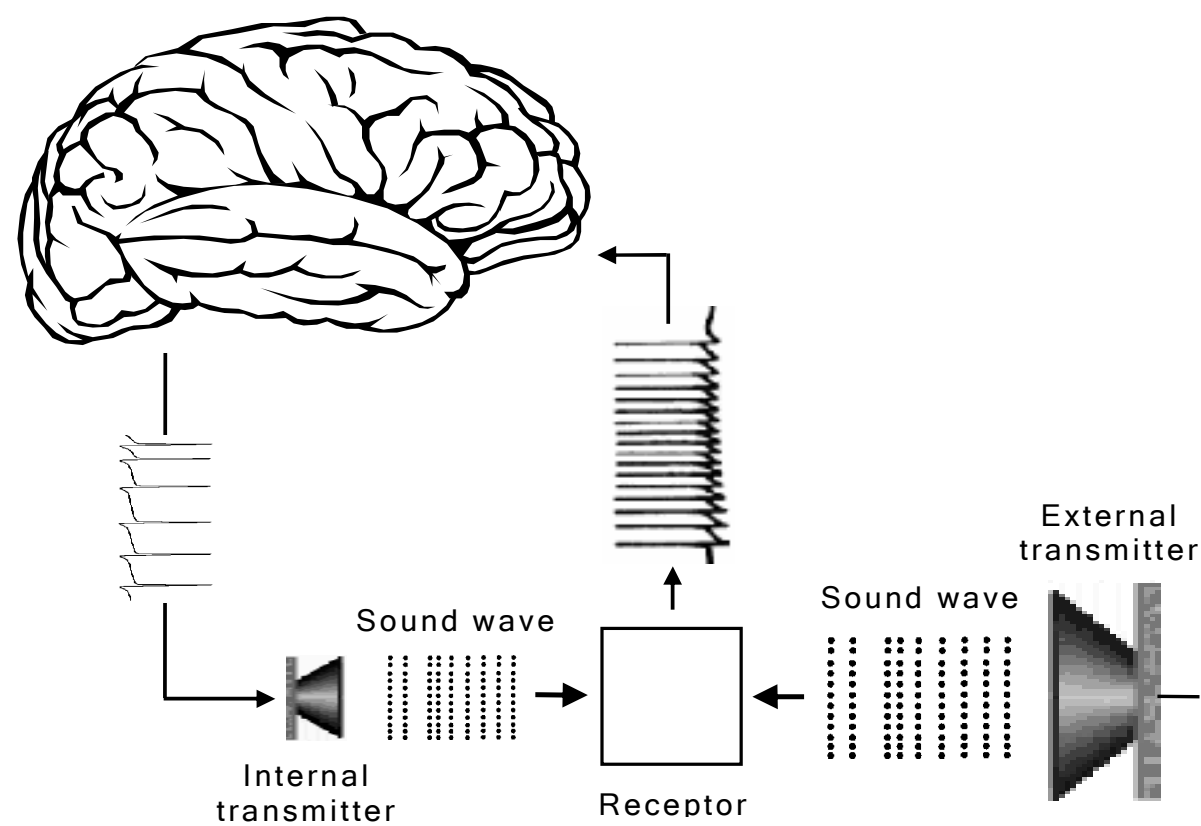

Fig. 1. The behavior of the perceptual loop in the Entropy Theory. The brain controls an internal transmitter from which internally generated stimuli, here imagined as longitudinal sound pressure waves, impinge upon the receptor. External stimuli (here, the sound pressure waves) also impinge upon the receptor. From there, neural spikes travel to the brain. The brain sends back to the internal transmitter, for acknowledgment, the spikes evoked at the receptor by the internally generated stimuli. Norwich did not explain how the brain distinguishes spikes evoked by internal stimuli from spikes evoked by external stimuli.

For hearing, there "will be observed to be a generator of audible sound" [3, p836] (also [4], [5]), which may explain the ear's known emission of narrowband acoustic signals [4], [5], the spontaneous otoacoustic emissions (SOAEs; e.g. [25]). Norwich [5] states that the latter define the auditory continuum that can be perceived, and that they play a role in the auditory perceptual loop (see also [4]).

\section{Why a Loop?}

In the Entropy Theory's model of perceptual foreknowledge, the generation of self-calibration signals of differing intensities by internal transmitters is hypothetically controlled by the brain, as follows. The receptor hypothetically collects both internal and external signals [3]-[5]. (Norwich uses "signal" and "stimulus" interchangeably.) Norwich states that "The entropy-establishing process begins with the generation of a [internal] sensory signal [i.e. stimulus] by the stimulus generator. This is followed by receipt of the [external] stimulus by the sensory receptor, transmission of action potentials by the sensory neurons, and finally recapture of the [response to the internal] signal by the generator" $[4$, p187; bracketed terms supplied]. Hence "The capture of a signal [the receptor's response to the internally-emitted signal] by the generator after an appropriate interval of time identifies the [same] signal, to a degree of probability, with the [internally-emitted] signal originally generated" [4, p187; bracketed terms supplied]. That is, "Reverberation or echoing of the generated signal provides the evidence that the system is closed" $[4, p 184]$, i.e. that the signal was self-generated and therefore a calibration signal rather than an outside stimulus. The external signal does not echo [4].
Fig. 1 shows the perceptual loop.

\section{A Flaw In The Proposed Perceptual Loop}

There has been a vast accumulation of sensory research over the years since [3], which was the last of the three papers in which Norwich introduced the brain-receptor loop. The aforementioned mass of accumulated findings allows us to look for one crucial component of Norwich's "perceptual loop", namely, the "internal transmitters", the Entropy Theory's so-called sensory self-calibration mechanisms. It transpires that Norwich's putative candidates for the role do not do what he claimed. First, the ear's spontaneous otoacoustic emissions are one of a class of related emissions [26] which are artefacts of inner-ear mechanics, artefacts found in many but perhaps not all persons (see for example [27] and later reviews). These artefacts are not usually audible to the producer, although Norwich did not claim that they had to be. These emissions occur over only a restricted portion of the perceived range of frequencies, may be associated with auditory damage [27], and disappear after ingestion of aspirin [28], hardly the characteristics of a crucial mechanism. In vision, spontaneous isomerization of pigment molecules, so-called "dark light", does not involve the generation of visible light (e.g. [29]). The many years of work on cutaneous pressure and temperature receptors have not yet revealed warmth receptors that "must be capable of generating temperatures in excess of local skin temperature" [4, p180] or any self-generation of touch sensations, except perhaps in pathological cases. 




Fig. 2. The nesting of an infinity of perceptual loops, as necessitated by the anthropomorphization of the receptor in the Entropy Theory (see text). $\mathbf{I T}=$ internal transmitter; $\mathbf{R}=$ receptor.

The suggested self-generation of forces by muscle stretch receptors [5], or of odours or tastes, has not been found except in pathological cases.

Some possible reasons for the lack of Norwich's proposed "internal emitters" are pursued in the next section.

\section{DisCUSSION: THE ANTHROPOMORPHIZATION OF THE RECEPTOR}

Norwich [4, p186] stated that "It is taken as unconvincing that nature impregnates a receptor with innate uncertainty so that the receptor 'awakens' in doubt". Having dismissed outright the idea that "a receptor is in some way, designed structurally to gravitate towards the interrogative" [5, p537], Norwich continues "How, then, did the receptor become 'aware' of the existence of the array of potential stimulus magnitudes?" [5, p537]. His answer was the perceptual loop, including the receptor. To the latter, Norwich assigned "awareness" [5], [10], [17]. Regarding vision, Norwich suggested that "Suppose that we anthropomorphize the eye, bestow it with a memory and the ability to communicate its "thoughts", and ask it to discuss with us its impressions in the milliseconds following the appearance of the light..." [17, p157]. The chemosensory receptor was later also anthropomorphized, in a similar manner (see [10], p155). Regarding the "initial uncertainty", i.e. the earliest value of $E_{S}$ (see Eq. (2)) available to the receptor, Norwich states that "It seems to imply that the receptor was, in some way, 'aware of' or advised of the potential existence of stimuli which range over a whole continuum of magnitudes" [5, p537]. Norwich further states that "Clearly, it seems, the receptor has perceived all members of this array of stimuli in the past, and so retains some memory of the events" [5, p537].

Let us summarize the characteristics of the receptor according to the Entropy Theory. The receptor itself "perceives" (or "observes"; [30, p350], a behavior usually attributed to the whole organism. Further, the receptor has a "memory". Norwich [3, p836] noted that "Memory of the set of possible signals engenders doubt or uncertainty in the 'mind' of the receptor when confronted by an unknown signal". Thus, the receptor had a "mind", and could be "confronted". In the same paper, the word "mind" was used differently, to describe the perceptual loop itself: "a unit capable of doubting, or being uncertain ... in principle, a 
rudimentary 'mind" " ([3, p835]; also [14]). ${ }^{1}$ But a mind is undisputably a characteristic of the whole human, not a portion thereof.

If the receptor (or the perceptual loop) is indeed a mind or part of a mind, it must perforce contain its own perceptual loop - which must, by necessity, contain a receptor. That receptor (or the perceptual loop in which it is embedded) must contain its own perceptual loop, and so on ad infinitum. Such an infinity is not physically viable. First, it appears to need an infinite amount of energy. Even if the successively nested levels of receptors used successively lower amounts of energy, the total amount of energy required might still not sum to a finite value. Even if it did, there is no reason to believe that the amount of time needed by each nested receptor would differ. Thus the amount of time required to perceive any given stimulus would be infinite.

Fig. 2 shows the nesting of perceptual loops.

The empirical lack of internal emitters can be understood if we ask how the brain distinguishes spikes evoked by internal stimuli from spikes evoked by external stimuli. Norwich and co-authors never answered that question. Regardless, the sensory gamut appears in a continuous flow, except in the most highly contrived laboratory conditions. But Norwich and co-authors conceived of perception as an "act", perhaps a purposeful one, having a "before", a "during", and an "after". That division is presumably what led Norwich to state that the internal signal is generated by the internal emitter "before" the external [stimulus] signal arrives at the receptor. This division of the stimulus gamut into "events" was necessary in order to apply information theory. Such a concept might be attractive to some, if, say, all stimulation occurred in discrete packets long enough for the receptor to reduce its uncertainty, followed by time gaps long enough for uncertainty to be reestablished. But the Entropy Theory gives no hint as to how that would be done.

In real life, stimuli tend to occur in a quasi-continuous stream having changeable characteristics. This begs the question of what exactly is to be considered "the stimulus", and when exactly are "before" and "after". Faced with uncertainty about the what and the when of the stimulus stream, the emission of calibration stimuli by the internal emitters would have to be continuous, producing a ruinous drain of energy.

\section{CONCLUSIONS: THE PERCEPTUAL LoOP Stems From A NEEDLESS PARADOX}

The literature shows that the self-calibration mechanisms called for in the Entropy Theory are unlikely to exist. Further, the "perceptual loop" that allegedly uses those mechanisms is unphysical. In the Entropy Theory the receptor requires foreknowledge, a situation forced by the philosophical basis of the Entropy Theory: "Perception only if uncertainty, but uncertainty only if perception" [5, p538; also p537], a statement which Norwich himself admits is a paradox [e.g. 3, p835]. That paradox seems needless, and has not been used by

\footnotetext{
${ }^{1}$ The amused reader may note that in one paper Norwich referred to both the "mind's eye" and the "eye's mind" [17].
}

other investigators. Indeed, the need for foreknowledge led to confusing complications. For example, it is unclear why Norwich mentions the conscious holding of different weights as a way that the receptor learns and remembers the range of available weights [4], after having already invoked the need [5] for a "muscular component which is capable of actively generating a force" in order to achieve the same goal. In any case, receptors do not learn and do not have memories; learning and memory are universally accepted as central processes. There is an obvious alternative approach: to postulate that perception has nothing at all to do with reduction of Shannon entropy (uncertainty), the core of the Entropy Theory, and to consider other general sensory models, of which there are plenty.

\section{ACKNOWLEDGMENT}

Work commenced at Dept. of Psychology, University of Toronto in Mississauga. Self-funded. L. Nizami thanks Professor Claire S. Barnes for insightful comments made during careful proofreading.

\section{REFERENCES}

[1] K.H. Norwich, "Information, memory, and perception," Inst. Biomed. Eng. U. Toronto vol. 17, 1975.

[2] K. H. Norwich and E. Sagi, "Deriving the loudness exponent from categorical judgments,” Percept. Psychophys., vol. 64, pp. 804-814, 2002.

[3] K. H. Norwich, "Why the eye may be found to be a source of light," Proc. $6^{\text {th }}$ Int. Cong. Cyber. Syst., World Org. Gen. Syst. Cyber., vol. 2, pp. 831-836, 1984.

[4] K. H. Norwich, "To perceive is to doubt: the relativity of perception," J. Theor. Bio., vol. 102, pp. 175-190, 1983.

[5] K. H. Norwich, "Perception as an active process," Math. Comput. Sim., vol. 24, pp. 535-539, 1982.

[6] K. H. Norwich, "The magical number seven: making a 'bit' of 'sense'," Percept. Psychophys., vol. 29, pp. 409-422, 1981.

[7] K. H. Norwich and W. Wong, "Sensory function in extraterrestrial beings," Ann. Fond. Louis de Broglie, vol. 22, pp. 161-168, 1997.

[8] W. Wong and S. Figueiredo, "On the Role of Information and Uncertainty in Auditory Thresholds," Proc. 2002 Int. Conf. Auditory Display, Int. Comm. Aud. Display (ICAD), pp. ICAD02-1 - ICAD02-6, 2002.

[9] C. E. Shannon, "A mathematical theory of communication," Bell Syst. Tech. J., vol. 27, pp. 623-656, 1948.

[10] K. H. Norwich, "Toward the unification of the laws of sensation: some food for thought," in Sensory Science Theory and Applications in Foods, H. T. Lawless and B. P. Klein, Eds. New York: Marcel Dekker Inc., 1991, pp. 151-183.

[11] K. H. Norwich, "On the fundamental nature of perception," Acta Biotheor., vol. 39, pp. 81-90, 1991.

[12] K. H. Norwich, "Determination of saltiness from the laws of thermodynamics - estimating the gas constant from psychophysical experiments," Chem. Senses, vol. 26, pp. 1015-1022, 2001.

[13] K. H. Norwich, "Physical entropy and the senses," Acta Biotheor., vol. 53, pp. $167-180,2005$.

[14] K. H. Norwich and K. M. V. McConville, "An informational approach to sensory adaptation,” J. Comp. Physiol., vol. A168, pp. 151-157, 1991.

[15] K. H. Norwich, Information, Sensation, and Perception. Toronto: Academic Press, 1993.

[16] K. H. Norwich, "An hypothesis on the processing of information by sensory receptors," Dig. 11th Int. Conf. Med. Biol. Eng., The Conference Committee, pp. 610-611, 1976. 
[17] K. H. Norwich, "An hypothesis on information, memory, and perception," Med. Hyp. vol. 4, pp. 156-164, 1978.

[18] K. H. Norwich, "Uncertainty in physiology and physics," Bull. Math. Biol., vol. 43, pp. 141-149, 1981.

[19] K. H. Norwich, "The psychophysics of taste from the entropy of the stimulus," Percept. Psychophys., vol. 35, pp. 269-278, 1984.

[20] K. H. Norwich, "On the information received by sensory receptors," Bull. Math. Biol., vol. 39, pp. 453-461, 1977.

[21] K. H. Norwich, "Do sensory receptors express their state of certitude?" Psychonom. Soc. Abstracts, vol. 25, p. 24, 1984.

[22] W. Wong and K. H. Norwich, "Simulation of human sensory performance," BioSystems, vol. 43, pp. 189-197, 1997.

[23] K. H. Norwich, "On the theory of Weber fractions," Percept. Psychophys., vol. 42, pp. 286-298, 1987.

[24] W. R. Garner and H. W. Hake, "The amount of information in absolute judgments," Psych. Rev., vol. 58, pp. 445-459, 1951.
[25] P. M. Zurek, "Spontaneous narrow-band acoustic signals emitted by human ears," J. Acoust. Soc. Am., vol. 69, pp. 514-523, 1981.

[26] E. Zwicker and E. Schloth, "Interrelation of different oto-acoustic emissions,” J. Acoust. Soc. Am., vol. 75, pp. 1148-1154, 1984.

[27] P. M. Zurek, "Acoustic emissions from the ear: a summary of results from humans and animals," J. Acoust. Soc. Am., vol. 78, pp. 340-344, 1985.

[28] D. McFadden and H. S. Plattsmier, "Aspirin abolishes spontaneous otoacoustic emissions," J. Acoust. Soc. Am., vol. 76, pp. 443-448, 1984.

[29] R. B. Barlow, R. R. Birge, E. Kaplan, E., and J. R. Tallent, "On the molecular origin of photoreceptor noise," Nature, vol. 366, pp. 64-66, 1993.

[30] K. H. Norwich, C. N. L. Seburn, and E. Axelrad, "An informational approach to reaction times," Bull. Math. Biol., vol. 51, pp. 347-358, 1989. 